\title{
EDITORIAL
}

\section{THE BENEFITS OF INTERNATIONAL UNIVERSITY PARTNERSHIPS: THE PERSPECTIVES OF A CANADIAN FACULTY MEMBER}

In November 2013, I had the privilege of visiting the Escola de Enfermagem, Universidade Federal do Rio Grande do Sul (UFRGS) in Brazil. During my nine day visit, I met with faculty members and graduate students, conducted two graduate classes on health systems integration, and visited various healthcare facilities. This visit had a significant impact on me professionally and personally. International partnerships in healthcare between universities from various countries have distinct benefits. I would like to highlight five.

1) Exposure to other healthcare practices, organizations and systems. Healthcare practices and organizations are influenced by social, cultural, political, and economic contextual factors ${ }^{(1,2)}$. These vary from one country to another, and also within countries and settings. Contextual factors impact us personally but also help us begin to understand how these factors impact and affect our understanding of health and healthcare. Furthermore it provides understanding of the needs of different populations and how health services are delivered in varying contexts.

2) Introduction to nursing education in other countries. International partnerships facilitate sharing of nursing knowledge ${ }^{(2)}$, particularly around nursing education. Reviewing different curriculums and educational approaches is extremely valuable when considering nursing competencies, teaching approaches, and curriculum revision. For example, understanding how research is taught and included in UFRGS' undergraduate program has influenced my thinking of how research may be presented in our new undergraduate curriculum currently being developed at the University of British Columbia, Okanagan Campus.

3) International study opportunities for students and post-doctoral fellows. A key benefit of an international university partnership is the opportunity for graduate and undergraduate students and post-doctoral fellows to study abroad. Again, exposure to different healthcare settings and practices will help students to be more flexible in their learning environments. It will help them to better understand the socio, cultural, political, and economic components of health and healthcare. Furthermore, it builds capacity in students in a variety of areas such as nursing knowledge and research methodology. The Brazilian government actively facilitates such study for nursing students and post-doctoral fellows (e.g., sandwich programs). Providing similar opportunities for Canadian nursing students would also be beneficial.

4) Collaborative research. Collaborative research builds capacity in research team members, research staff, and students working on research projects. Joint research can maximize scarce resources more effectively and facilitate greater generalizability of results.

5) Learning from each other. Of greatest importance is learning from each other. International university partnerships need to ensure there is mutual benefit; benefit must go beyond the historical north-south flow of information and focus on what we can learn from each ${ }^{(2)}$. Learning will occur at various levels (e.g., administration, faculty, students) and in numerous areas (e.g., differences in culture and context, research, education). Where the cross-learning can be of greatest value is in more effective and efficient care and ultimately the improvement of patient outcomes.

International partnerships are exceedingly valuable. Clearly established objectives, roles, and accountabilities, and regular communication are essential to ensure mutual benefit for all stakeholders. Although not always easy, international partnerships hold significant rewards for those participating.

a PhD RN, Assistant Professor, School of Nursing, Faculty of Health and Social Development, University of British Columbia, Okanagan 


\section{EDITORIAL}

\section{REFERENCES}

1 Muula A. Goodbye collaboration, welcome partnerships in international research? Croat Med J. 2010; 51:35960.

2 Scarr E, Pulcini J, Makonnen J, Turk K, Wheeler K, Eissler LA, Schumann L, et al. International nursing partnerships. Nurse Pract. 2012; 37(6):11-2. 